\title{
REPRESENTACIONES SOCIALES DE EMPLEO Y DESEMPLEO EN ESTUDIANTES UNIVERSITARIOS DE QUITO, ECUADOR
}

\author{
SOCIAL REPRESENTATIONS ABOUT EMPLOYMENT AND \\ UNEMPLOYMENT OF UNIVERSITY STUDENTS FROM QUITO, \\ ECUADOR
}

\author{
Teresa Margarita Torres López* \\ Jazmín Aranzazú Munguía-Cortés** \\ María Maura Torres-Valdovinos****
}

Fecha de recepción: 2 de marzo de 2018 - Fecha de aceptación: 24 de mayo de 2018

\section{Resumen}

\begin{abstract}
El objetivo de este estudio es analizar las representaciones sociales (RS) de empleo y desempleo en estudiantes universitarios de Quito, Ecuador. Se utilizó la técnica asociativa de listados libres para conocer el contenido y estructura de las RS, así como la identificación del núcleo central y los elementos periféricos. Para quienes participan el empleo es una ocupación y un derecho que se realiza a cambio de un salario, requiere de experiencia, conocimientos, compromiso, esfuerzo, motivación, responsabilidad, deseos de superación, además de contar con suerte para encontrarlo. Genera estabilidad (emocional y económica), bienestar, autoridad, desarrollo, dignidad e independencia; así como las posibilidades para su futuro con respecto a éxitos y logros. En forma negativa implica inversión de tiempo, puede ser agotador y generar insatisfacción, sobre todo cuando no es acorde a sus necesidades y expectativas. Además, se le asocia al desempleo. El desempleo representaría un tiempo libre, desocupado y desperdiciado que genera pobreza, deudas y precariedad. En el ámbito personal produce inestabilidad, dependencia, falta de desarrollo, insatisfacción de necesidades; emociones negativas como ansiedad, depresión, desesperación y angustia. En lo familiar, puede provocar problemas como divorcios y falta de estudio en las hijas y los hijos; en lo social, vagancia y suicidio. Además, hay pocas oportunidades de empleo para la población estudiantil. Las personas participantes establecen, como una de sus conclusiones elementales, que las formas de afrontamiento al mismo son buscar empleo con esfuerzo y humildad, así como migrar a otros países. Las RS sobre los conceptos estudiados muestran una visión compleja y holística, ya que no sólo se incluyen elementos de tipo económico sino también los efectos personales y sociales que la problemática implica.

Palabras clave: Empleo de jóvenes; Desempleo de los jóvenes; Trabajo; Educación y empleo; Conoci-
\end{abstract} mientos tradicionales

\section{Abstract}

The objective of this study is to analyze the social representations (SR) of employment and unemployment of college students from Quito, Ecuador. The associative technique of free lists was used to know the

*_Universidad de Guadalajara, México, Profesora-Investigadora, tere.torres.cucs@gmail.com

** Universidad de Guadalajara, México, Profesora-Investigadora, Asociada del Departamento de Psicología Aplicada, jazminmunguia@gmail.com

*** Universidad de Guadalajara, México, Profesora de Carrera Asociada de la Escuela Preparatoria Regional de Ciudad Guzmán, Módulo Tecalitlán,maura_tv@hotmail.com 
content and structure of the $S R$, as well as the identification of the central nucleus and the peripheral elements. For the participants, employment is an occupation and a right that is carried out in exchange for a salary, requires experience, knowledge, commitment, effort, motivation, responsibility, as well as the possibility to find it. It generates emotional and economic stability, well-being, authority, development, dignity, and personal independence along with a promising future and successes. Sometimes, in a negative way, when it does not meet one's needs and expectations, it can be exhausting and dissatisfying. Unemployment is defined as a situation where someone of working age is not able to get a job; it generates poverty and debt. It also produces psychological instability, dependence, lack of personal development, and negative emotions such as anxiety and depression. In addition, it causes family problems such as divorce and lack of study opportunities in children. Moreover, there are few open positions for students. The participants states, as one of its elementary conclusions that there are two ways of coping with unemployment: to seek employment with effort and humility, or to emigrate. The SR on the concepts studied show a complex and holistic view, since not only elements of economic type are included but also the personal and social effects that the problem implies.

Key Words: Youth employment; Youth unemployment; Labour; education and employment; Traditional knowledge

\section{Introducción}

En la actualidad, la forma social del empleo actual y la nueva organización del trabajo se caracteriza por la incertidumbre en contratación, la desindustrialización, el aumento del desempleo, la precariedad, la flexibilidad y el incremento de la informalización laboral (Pulido-Martínez, \& CarvajalMarín, 2013). Las condiciones laborales son cada vez más flexibles y precarias, con contratos a tiempo parcial, por tiempo determinado o temporal, subcontrataciones, entre otras modalidades similar talante (Nascimento, \& Álvaro, 2014; Carballo-Villagra, 2005). Así la precariedad laboral, o aún más la falta de un empleo, no sólo merma el acceso a las diversas fuentes de obtención de recursos, sino que genera una serie de aspectos negativos sobre el bienestar psicológico de las personas que lo sufren. Estar sin empleo se convierte en un obstáculo que dificulta el potencial desarrollo de los individuos (Izquierdo, \& Alonso, 2014). A lo anterior se aúna la inseguridad laboral es decir, la incapacidad percibida para mantener la continuidad laboral ante una situación de amenaza del empleo, lo cual depende de la formación para el trabajo, la empleabilidad en el mercado laboral y el subempleo (Sora, Caballer, \& Peiró, 2014).

De partida cabe diferenciar dos conceptos clave utilizados en este estudio: trabajo y empleo. De acuerdo al Tesauro de la Organización Internacional del Trabajo (es una compilación de más de 4000 términos relacionados con el mundo del trabajo, en inglés, francés y español) el primero se define como el conjunto de actividades humanas, remuneradas o no, que producen bienes o servicios en una economía, o que satisfacen las necesidades de una comunidad o proveen los medios de sustento necesarios para los individuos. Empleo es trabajo efectuado a cambio de pago. También se refiere al número de personas bajo un régimen de autoempleo o empleo remunerado (Oficina Internacional del Trabajo [OIT], 2018).

Considerando dichos conceptos, la presente propuesta incluye la visión de los futuros trabajadores y de las futuras trabajadoras a partir de estudiar los procesos subjetivos compartidos que se manifiestan a través de sus discursos y prácticas, en el marco de la cultura en que viven. Las representaciones que éstos le otorgan al empleo así como las atribuciones e imágenes que permitirán acceder a su mundo de significados, así como a sus propuestas ante la problemática en torno a su inserción laboral.

Una perspectiva teórica y metodológica que nos permite un acercamiento al conocimiento del empleo y el desempleo dentro de un marco cultural son las representaciones sociales (RS). Jodelet (2011) indica que son una forma específica de conocimiento, el conocimiento ordinario, incluido en la categoría del sentido común y que tiene como particularidad la de ser socialmente construido y compartido en el seno de diferentes grupos. Esta forma de conocimiento tiene una raíz y un objetivo práctico: apoyándose 
en la experiencia de las personas, sirve de lectura de la realidad y de guía de acción en la vida práctica y cotidiana. De acuerdo con Sibaja (2013) las RS circulan por medio de los discursos y cobran vida a través de las prácticas discursivas de la cotidianidad. Son compartidas, más no son inmutables, ya que son susceptibles al cambio gracias al intercambio individual, puesto que los individuos las construyen.

Existen dos enfoques al estudio de las RS: el estructural y el procesual. En el primero, los contenidos de las RS son considerados como estructuras organizadas, se caracteriza por identificar su estructura o su núcleo y por desarrollar explicaciones acerca de las funciones de esta estructura (Abric, 2001). Su énfasis es el estudio del núcleo central o sistema que da a las RS el significado, y del periférico, que comprende los elementos de las RS, que están influenciados por el contexto social inmediato en el que los sujetos viven. El enfoque procesual identifica a las RS como procesos discursivos, caracteriza al ser humano como productor de sentidos y focaliza su análisis en las producciones de significados del lenguaje, a través de los cuales los seres humanos construyen su mundo (Banchs, 2000). Ambos enfoques son considerados para esta investigación.

Se cuenta con algunos estudios sobre el empleo y el desempleo con base en el enfoque teórico metodológico de las RS.

En México el estudio de Torres-López, Acosta, y Aguilera (2017) analiza el caso de los jóvenes estudiantes de bachillerato sin trayectoria laboral. Las RS de empleo son las de una ocupación que requiere contar con los elementos necesarios tanto de preparación académica, como de valores y esfuerzo personales, lo que derivará en la obtención de un ingreso económico y de bienes materiales, además de beneficios en el ámbito personal. Implica también algunos aspectos negativos, como el tiempo ocupado, la rutina y el cansancio. Las RS de desempleo son de un tiempo desocupado que implica la falta de dinero y la vivencia de pobreza, con efectos personales, sociales que generan emociones negativas. Las RS del empleo y desempleo muestran una serie de expectativas que difícilmente llegarán a coincidir con la realidad a la que se enfrentarán los jóvenes en su proceso de inserción laboral, máxime cuando las opciones que se presentan en el contexto mexicano, en torno a la preparación para el empleo, son limitadas.

Otro estudio (Torres-López, \& Díaz-Villanueva, 2016) identifica las RS de empleo y desempleo de estudiantes universitarios. Para las personas participantes, el empleo es una ocupación o actividad que requiere de aptitudes, actitudes y energía personales para su realización; comprende diversos componentes de tipo económico, genera beneficios materiales (producto de la percepción económica), más beneficios personales y sociales. Las RS de desempleo implican la falta de empleo y de oportunidades con implicaciones de escasez económica, dentro de un contexto nacional crítico, con efectos en lo personal y social; genera además emociones negativas. Las RS de empleo y desempleo parecen mostrar una relación complementaria, en ambos conceptos se incluyeron categorías coincidentes, así como elementos de tipo negativo en empleo (precariedad) y de tipo positivo en desempleo (afrontamiento). Ello indica que ambas RS se alimentan tanto de las expectativas de quienes aún no desarrollan actividades laborales remuneradas, como con las experiencias de quienes ya tienen empleo (que no han sido del todo positivas).

Asimismo, un estudio sobre experiencias y RS del empleo en jóvenes con licenciatura terminada que se encontraban en situación de desempleo o subempleo, se realizó por Navarro (2007) en la ciudad de México. Los resultados muestran que el empleo es difícil de encontrar, consideran un logro obtenerlo y es un medio para alcanzar otros fines como lograr la independencia (residencial y económica). Además, tener una carrera no asegura el ingreso laboral y la experiencia cuenta más que los estudios al momento de ser seleccionado para un puesto de trabajo. Por ello, algunos están dispuestos a sacrificar sueldo o condiciones laborales con tal de conseguir esa experiencia deseada, sobre todo quienes cuentan con apoyo familiar económico directo y quienes tienen menos tiempo de haber egresado.

En cuanto a otros estudios realizados en Latinoamérica uno fue con jóvenes varones pobres y ocupados en condiciones de precariedad de Buenos Aires. Para los participantes el trabajo es visto como una necesidad intrínseca, y una forma de realización personal. El trabajo los ha emancipado 
desde pequeños, los ha llevado a la autonomía y es sinónimo de independencia. Dentro de los aspectos negativos que perciben del empleo es considerarlo un obstáculo a su sociabilidad. También, los horarios y la duración de la jornada de trabajo les afectan: ocasiona problemas familiares, les impide ver a sus hijos, estar con sus amistades y afecta su participación en organizaciones sociales. Por tanto, el empleo les quita tiempo para extender sus vínculos o afianzarlos (Longo, 2003).

Otro estudio en Latinoamérica, incluyó jóvenes universitarios con empleo remunerado de Venezuela. Para las y los participantes las RS del empleo destacan la posibilidad de satisfacer las necesidades económicas y familiares, además de la valoración del trabajo a pesar de esfuerzo que implica tener que estudiar y trabajar. Para los participantes el trabajo es una forma de vida, asumida como una rutina que permite satisfacer sus necesidades fundamentales. Se concluye que el trabajo tiene un valor instrumental al ser un medio para el logro de múltiples fines, alcanzar ingresos y satisfacer necesidades. Así como un valor expresivo, con la necesidad de ser independiente, autónomo, autosuficiente y asumir responsabilidades de adultos. Sin embargo, las personas participantes no mostraron claridad en cuanto al trabajo productivo decente, como es la conciencia de los derechos laborales fundamentales, la seguridad social y otros aspectos legales (Pereira, 2013).

A partir de lo expuesto el objetivo de este estudio es analizar las representaciones sociales (RS) de los conceptos de empleo y desempleo en estudiantes universitarios de Quito, Ecuador, a fin de contar con más elementos para planear, implementar y evaluar programas de preparación y promoción para el empleo.

\section{Metodología}

\section{Diseño}

El diseño de estudio comprendió tres fases: a) la obtención de información para identificar el contenido de las RS con base en el análisis procesual cualitativo e incluyó análisis de categorías temáticas emergidas del discurso de los participantes (Banchs, 2000); b) la verificación de la centralidad de las RS a través de la identificar del nodo central empleando el criterio de saliencia con base en el análisis prototípico (Verges, Tyszka, \& Vergès, 1994), y c) la descripción de la organización y la estructura de las RS, con base en el índice de distancia entre las palabras incluidas (Abric, 2001).

\section{Participantes}

El estudio se realizó en Quito, Ecuador. Se integró una muestra de tipo propositivo (Suri, 2011). Los criterios para su selección fueron: estudiantes universitarios, hombres y mujeres, mayores de edad y habitantes de la ciudad citada. Todos fueron abordados en las instalaciones de una universidad pública.

Participaron 80 personas (47 mujeres y 33 hombres), sus edades fluctuaron entre los 21 y los 33 años (con un promedio de 22.9 años), su nivel de estudios fue universitario, 10 contaban con empleo remunerado (el cual desarrollaban desde uno hasta cinco años atrás). Todos se identificaron de nacionalidad ecuatoriana.

\section{Instrumentos}

Las técnicas de recolección de datos fueron dos:

a) Técnica asociativa de listados libres, que consiste en pedir a los informantes términos relacionados con una esfera conceptual particular: "Dime cinco palabras o frases que se te vienen la mente cuándo escuchas: empleo/desempleo" después se les pide una pequeña explicación de por qué dijeron 
cada una de ellas. El tiempo de aplicación fue de máximo cinco minutos a 10 minutos, lo que facilitó la participación de las personas. Favorece la expresión espontánea de las personas participantes y por ello se espera que el contenido evocado esté más libre de racionalizaciones, sesgos de defensa o deseabilidad social (Ruiz, Ponce de León, \& Herrera, 2001).

b) Cuestionario de comparación de pares: con las respuestas más frecuentes se elaboraron dos cuestionarios de comparación de pares: uno para el concepto de empleo y otro para el desempleo. Este segundo instrumento permitió a las personas entrevistadas calificar los términos seleccionados. Ello mediante la asociación de los dos términos que a su juicio consideran que son los más característicos de empleo (y desempleo) e hicieran una marca (con una letra A) en ellos. De los elementos restantes, eligieran dos elementos que a su juicio consideran que son los menos característicos de empleo (y desempleo) y los marcaran (con una letra B). Se continuó hasta que realizaron marcas a los diez términos (con letras C, D y E). Así al final se cuenta con cinco pares de asociaciones (Abric, 2001).

\section{Procedimiento}

Antes de cada entrevista se explicaron los objetivos del estudio y se obtuvo el consentimiento informado verbal (Aguilera-Guzmán, Mondragón, y Medina-Mora, 2008), garantizándole a cada participante la confidencialidad y el anonimato en la recolección y el análisis de la información. La participación de las personas entrevistadas fue voluntaria. El presente estudio fue evaluado y aprobado por el Comité de ética, investigación y bioseguridad del Centro Universitario de Ciencias de la Salud, de la Universidad de Guadalajara, México (registro no. CI02916).

\section{Análisis de datos}

Se realizaron tres tipos de análisis:

a) Análisis procesual: con base en las palabras obtenidas en los listados libres se realizó una categorización de las definiciones dadas para cada palabra, lo cual se hizo con análisis de contenido temático (Minayo, 1995) con el apoyo del software Atlas.Ti.

b) Análisis prototípico: con base en las palabras obtenidas en los listados libres se identificó la relevancia (salience) de cada término a través del índice de $\operatorname{Smith}^{1}$ (1993); este cálculo se basa tanto en la frecuencia de mención del término como en el rango de la ubicación que le otorgó cada informante a cada palabra (de tal forma que los primeros mencionados en el listado tienen un mayor peso que los últimos). Para ello se utilizó el software Anthropac v. 4.98. Se procedió a clasificarlos considerando la frecuencia y el rango promedio obtenido en los dos grupos de participantes. Las palabras que constituyen el núcleo central de las RS serían aquellas que son citadas más veces y en primer lugar (rango) (Ruiz, et al., 2001).

c) Luego de obtener las listas de palabras, se tabularon los términos por frecuencia de mención. Se escogieron diez palabras de cada lista para generar el instrumento de cuestionario de pares aplicado en la segunda fase del estudio, uno para empleo y otro para desempleo. Los datos obtenidos en los cuestionarios de comparación de pares fueron analizados por medio de la obtención del índice distancia, que evalúa la relación de similitud y la relación de antagonismo o de exclusión. Además, mide la intensidad de la diferencia observada entre dos elementos en una población dada de tal forma que +1 es la similitud y -1 la exclusión máxima. Para ello, se asignó un valor numérico a los pares de palabras seleccionadas. La escala de evaluación fue de 0 a +2 con relación a la importancia asignada a la asociación $(\mathrm{A}=+2, \mathrm{~B}=-2, \mathrm{C}=+1, \mathrm{D}=-1$ y $\mathrm{E}=0$ ) (Abric, 2001). A partir de estas

1 Por cada listado libre individual se calcula:

$\mathrm{s} j=1 \underline{\mathrm{r} j} 1 \quad \mathrm{~s} j=\underline{\mathrm{n} r} \mathbf{r}$

$\mathrm{n} 1 \mathrm{n} 1$

Donde $r_{j}=$ posición del ítem $j$ en la lista, y $\mathrm{n}=$ número de ítems de la lista. Para obtener el índice de salience, es necesario obtener el promedio de $s_{j}$ de todos los respondientes (Smith, 1993). 
puntuaciones se calculó el análisis de distancia ${ }^{2}$ con el apoyo de una hoja de cálculo de Excel. Con los índices obtenidos en la relación de cada uno de los pares seleccionados se construye un grafo. Esto es, una representación gráfica de la conexión de todas las palabras. El procedimiento para su construcción proviene de la Teoría de grafos y es descrita por Doise, Clemence y Lorenzi-Cioldi (1993). El estudio de las asociaciones identificadas permite analizar las relaciones significativas de la naturaleza de la representación de las personas o de ciertas dimensiones de esta representación (Abric, 2001).

Confiabilidad y validez de la información. Se realizó triangulación por contrastación teórica y de investigadores (Flick, 2014), ya que en el proceso de análisis participaron todos los investigadores en forma separada, los que llegaron a los mismos resultados.

\section{Resultados}

\section{Empleo}

\section{Análisis procesual: El contenido de las RS de empleo}

En la Tabla 1 se presentan las categorías encontradas en el análisis de las palabras obtenidas en los listados libres para las RS de empleo.

Tabla 1

Categorías encontradas en el análisis de las palabras obtenidas en los listados libres para las RS de empleo

\begin{tabular}{|c|c|c|c|}
\hline Categoría & Definición & Frecuencia & $(\%)$ \\
\hline Componentes de empleo & Términos asociados al desarrollo de una actividad laboral & 28 & 27.0 \\
\hline Elementos necesarios & Requisitos para la realización de actividades en un empleo & 24 & 23.0 \\
\hline Beneficios personales & Consecuencias positivas para la persona que realiza el trabajo & 21 & 20.1 \\
\hline Aspectos económicos & $\begin{array}{l}\text { Obtención de un salario para cubrir todo tipo de necesidades } \\
\text { económicas }\end{array}$ & 19 & 18.3 \\
\hline Aspectos negativos & Derivados de la realización de un determinado empleo & 10 & 9.6 \\
\hline Beneficios materiales & Adquisiciones de bienes específicos producto del salario laboral & 2 & 2.0 \\
\hline Total & & 104 & $100 \%$ \\
\hline
\end{tabular}

Fuente: Elaboración de los autores.

Las categorías que incluyen más términos fueron los componentes de empleo (ocupación, trabajo, empleador, organización, empresa, etc.), elementos necesarios (experiencia, motivación, responsabilidad, suerte, etc.) y beneficios personales (futuro, estabilidad, bienestar, independencia, etc.). La categoría de componentes del empleo incluyó sinónimos (trabajo, ocupación, un joven indicó: "permite que la persona se desarrolle en el área laboral permitiendo un intercambio remunerativo por la realización de la tarea"). Elementos de una organización (empleador, empresa, trabajador, organización

2 La fórmula utilizada para su obtención es:

$\mathrm{D}=[2$ (en - sumatoria dj) $)]-1, \mathrm{D}=$ distancia observada, $\mathrm{n}=$ número de sujetos, $\mathrm{e}=$ escala 
y uniforme), un antónimo (desempleo) y la palabra derecho ("todos podemos ejercer libremente el trabajar, sin que nada de índole legal lo prohíba"). En la categoría de elementos necesarios se incluyeron aspectos personales, (responsabilidad, compromiso, esfuerzo: "requiere del trabajo de las personas y el sacrificio para realizarlo"), motivaciones (deseos de superación, gusto: "es importante realizar tareas que sean agradables y afines a todo lo que he estudiado, ya que así serán realizadas de mejor manera"), académicos (conocimientos, experiencia: "el laborar y realizar las diferentes actividades nos ayuda a aprender muchas cosas, el conocer nuevas estrategias") y externos como suerte ("es cuestión de tener una palanca o alguien asertivo de la organización para que pueda colaborar"). Y en la categoría de beneficios personales se citaron efectos emocionales (tranquilidad, satisfacción: "puedo cumplir con las metas personales y profesionales planificadas"), bienestar ("estabilidad emocional y económica para mi sustento diario"), superación (desarrollo, dignidad, independencia: "ya no dependería económicamente de mis padres, sino que yo administraría mi dinero"), material (comodidad, éxito, logros) y futuro ("trabajaré mi futuro con un empleo y me abriré oportunidades de crecimiento").

Las categorías con menos términos fueron en primer lugar los aspectos económicos (dinero, salario, etc.), y segundo lugar, los aspectos negativos. Para las personas participantes el empleo implica tiempo (“ocupar un espacio que tenías libre”), puede ser agotador, genera preocupación por no tener empleo y "porque el esfuerzo sea retribuido de la mejor manera y poder tener un ambiente adecuado sano y de bienestar", también insatisfacción al "no encontrar un empleo acorde a las expectativas planteadas" y es insuficiente, ya que "en nuestra realidad es difícil y escaso el empleo que vaya acorde a nuestras necesidades y conocimiento". Finalmente, la categoría de beneficios materiales "puedo comprar lo que necesito".

\section{Análisis prototípico: núcleo central y sistema periférico de las RS de empleo}

Se retuvieron las palabras que fueran evocadas por lo menos por dos personas. En el prototipo de las RS, los elementos hipotéticamente centrales son aquellos que son más frecuentes y evocados en los primeros lugares de la lista de palabras. En la Tabla 2 se presenta el prototipo (frecuencia x rango de evocación) de las RS del empleo.

Tabla 2

Prototipo (frecuencia x rango de evocación) de las RS de empleo

\begin{tabular}{|c|c|c|c|c|c|c|c|}
\hline & & Rango* & & & & & \\
\hline & & $<3.2$ Bajo & & & Alto $>3.2$ & & \\
\hline \multirow{15}{*}{ Frecuencia } & \multirow{7}{*}{$\begin{array}{l}\text { Alta } \\
>4\end{array}$} & Trabajo & 10 & (1.7) & Responsabilidad & 5 & $(4.0)$ \\
\hline & & Dinero & 9 & (2.4) & Desarrollo & 4 & $(4.25)$ \\
\hline & & Salario & 8 & $(2.7)$ & Motivación & 4 & $(4.0)$ \\
\hline & & Satisfacción & 5 & $(2.6)$ & & & \\
\hline & & Esfuerzo & 4 & $(2.5)$ & & & \\
\hline & & Conocimientos & 4 & $(2.75)$ & & & \\
\hline & & Ocupación & 4 & $(3.0)$ & & & \\
\hline & \multirow{8}{*}{$\begin{array}{l}\text { Baja } \\
<4\end{array}$} & Tiempo & 2 & $(2.5)$ & Experiencia & 3 & (3.3) \\
\hline & & Estabilidad & 3 & $(2.0)$ & Desempleo & 2 & $(4.5)$ \\
\hline & & Empleador & 2 & $(2.5)$ & Autoridad & 2 & (3.5) \\
\hline & & Derecho & 2 & $(3.0)$ & Bienestar & 2 & $(4.0)$ \\
\hline & & Necesidad & 2 & (1.0) & & & \\
\hline & & Insuficiente & 2 & $(2.5)$ & & & \\
\hline & & Economía & 2 & $(3.0)$ & & & \\
\hline & & Éxito & 2 & (3.0) & & & \\
\hline
\end{tabular}

*Rango promedio $3.280 .7 \%$ de evocaciones totales 
En las RS de empleo el rango de evocación retenido fue del $80.7 \%$, y el rango promedio fue de 3.2. Los términos ubicados en una frecuencia alta $(>4)$ y un rango bajo $(<3.2)$ son los que constituyeron el núcleo central de las RS de empleo: aspectos económicos (dinero, salario), términos asociados (trabajo, ocupación), requisitos (esfuerzo, conocimientos). Los términos ubicados en la segunda columna con frecuencia baja $(<4)$ y rango alto $(>3.2)$ son considerados parte del sistema periférico de las RS, se caracterizan por estar determinados por la historia individual de las personas y por sus experiencias particulares: aspectos asociados (desempleo), un requisito (experiencia) y efectos personales (bienestar, autoridad).

\section{Organización de las RS de empleo}

En la Figura 1 se muestra el grafo de las RS de empleo. Las palabras que obtuvieron mayor índice de distancia (es la suma de los índices ubicados en las uniones de las palabras) fueron Experiencia y Responsabilidad.

Figura 1

Grafo de la RS de empleo en Quito

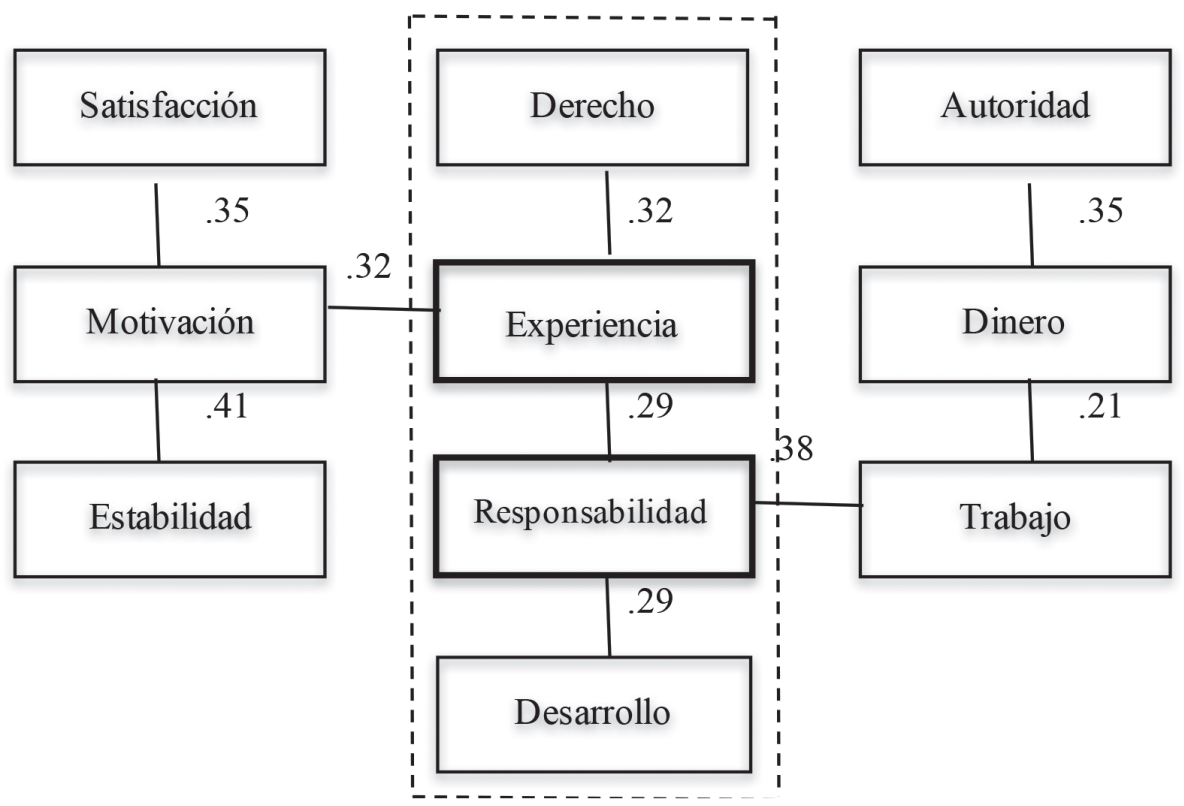

\section{Desempleo}

\section{Análisis procesual: el contenido de las RS de desempleo}

En la Tabla 3 se muestran las categorías encontradas en el análisis de las palabras obtenidas en los listados libres para las RS de desempleo, en los participantes. 
Tabla 3

Categorías encontradas en el análisis de las palabras obtenidas en los listados libres para las RS de desempleo

\begin{tabular}{lccc}
\hline Categorías & Definición & Frecuencia & $(\%)$ \\
\hline Componente económico & Sinónimos y otros términos asociados a la falta de dinero & 31 & 30.4 \\
\cline { 2 - 5 } Efectos personales & Consecuencias individuales por la falta de empleo & 21 & 20.6 \\
\cline { 2 - 5 } Elementos asociados & Términos relacionados a la falta de empleo & 18 & 17.6 \\
\cline { 2 - 5 } Emociones negativas & Efectos emocionales generados por el desempleo & 17 & 16.6 \\
Efectos sociales & Consecuencias del desempleo en la sociedad & 10 & 9.8 \\
nfrontamiento & Propuestas ante la falta de empleo & 5 & 5.0 \\
nyyly & & 102 & $100 \%$ \\
\hline
\end{tabular}

Fuente: Elaboración de los autores.

Las categorías que tienen mayor número de términos fueron el componente económico, los efectos personales y los elementos asociados. La de componente económico (pobreza, deudas, quiebra, etc.) implica según una persona participante que al no tener dinero "el estatus de vida disminuye, no se pueden satisfacer necesidades, provoca pobreza a nivel micro y macro", "los padres desempleados no pueden apoyar los estudios de sus hijos”. Además, genera insolvencia, deudas, quiebra y precariedad (“en el hogar falta el alimento, la vestimenta, la educación”). La categoría de efectos personales (intranquilidad, insatisfacción, problemas familiares, etc.) indicó que hay inestabilidad, dependencia, emociones como ansiedad e intranquilidad y falta de desarrollo ("el desempleo genera obstáculos para que una persona pueda desarrollarse, progresar y genera mediocridad", "existe estancamiento en las personas que se dedican a hacer solo funciones y no dan valor agregado a su trabajo") e insatisfacción ("ya que no se satisfacen las necesidades básicas”), problemas y dificultades. Así como desorganización familiar ("al verse en la necesidad caen en la desesperación, se comienzan a crear divorcios"). Y en la categoría de elementos asociados (desocupación, tiempo libre, pocas oportunidades, etc.), para las personas participantes el desempleo es un espacio de desocupación, tiempo libre y ocio ("como no trabajo, tengo mayor tiempo libre, que no es bien aprovechado, es tiempo desperdiciado"). Como estudiantes hay pocas oportunidades ("no existe mayor oportunidad de empleo para estudiantes de pregrado", "los horarios son complicados para poder conseguir empleo con la remuneración justa"). Es una realidad para el país que genera ansiedad y pesar en los pre-profesionales.

Las categorías con menos términos fueron las emociones negativas (depresión, desesperación, preocupación, angustia, etc.), los efectos sociales (vagancia, suicidio, inseguridad, abandono, etc.) y el afrontamiento al desempleo (migrar, búsqueda, esfuerzo, etc.). Los participantes señalaron al respecto la importancia de la búsqueda continua del empleo, con esfuerzo y humildad. Además de migrar, es decir, "buscar fuentes de empleo en otros países".

\section{Análisis prototípico: núcleo central y sistema periférico de las RS de desempleo}

Se retuvieron las palabras que fueran evocadas por lo menos por dos personas. En la Tabla 4 se presenta el prototipo (frecuencia x rango de evocación) de las RS de desempleo de los participantes. 
Tabla 4

Prototipo (frecuencia x rango de evocación) de las RS de desempleo

\begin{tabular}{|c|c|c|c|c|c|c|c|}
\hline & & Rango* & & & & & \\
\hline & & $<3.0$ Bajo & & & Alto $>3.0$ & & \\
\hline \multirow{14}{*}{ Frecuencia } & \multirow{5}{*}{$\begin{array}{l}\text { Alta } \\
>4\end{array}$} & Pobreza & 11 & $(2.5)$ & Insolvencia & 9 & $(3.1)$ \\
\hline & & Necesidades & 8 & $(2.75)$ & Problemas & 5 & (3.4) \\
\hline & & Inestabilidad & 4 & $(2.5)$ & Depresión & 5 & (4.4) \\
\hline & & Vagancia & 4 & $(1.75)$ & Insatisfacción & 4 & (3.2) \\
\hline & & Desocupación & 4 & $(2.0)$ & & & \\
\hline & \multirow{9}{*}{$\begin{array}{l}\text { Baja } \\
<4\end{array}$} & Pocas oportunidades & 3 & (3.0) & Tiempo libre & 3 & (5.0) \\
\hline & & Estrés & 3 & $(2.6)$ & Suicidio & 2 & $(5.0)$ \\
\hline & & Preocupación & 2 & $(1.0)$ & Desesperación & 2 & $(3.5)$ \\
\hline & & Realidad & 2 & $(3.0)$ & & & \\
\hline & & Trabajo & 2 & (3.0) & & & \\
\hline & & Ocio & 2 & (1.0) & & & \\
\hline & & Angustia & 2 & $(2.0)$ & & & \\
\hline & & Migración & 2 & (1.5) & & & \\
\hline & & Quiebra & 2 & $(3.0)$ & & & \\
\hline
\end{tabular}

En las RS de desempleo el rango de evocación retenido fue del $83.3 \%$, y el rango promedio fue de 3.0. Los términos ubicados en una frecuencia alta $(>4)$ y un rango bajo $(<3.0)$ son los que constituyen el núcleo central de las RS de desempleo. Se ubicaron en este espacio a términos de tipo económico (pobreza, necesidades), asociados (desocupación), efectos personales (inestabilidad) y un efecto social (vagancia). Los términos ubicados en la segunda columna con frecuencia baja $(<4)$ y rango alto $(>3.0)$ fueron considerados el sistema periférico de las RS, que sólo incluyó un término asociado (tiempo libre) y efectos personales (suicidio, desesperación).

Organización de las RS de empleo:

En la Figura 2 se muestra el grafo de las RS de desempleo. La palabra que obtuvo mayor índice de distancia (es suma de los índices ubicados en las uniones de las palabras) fue Insatisfacción.

Figura 2

Grafo de la RS de desempleo en Quito

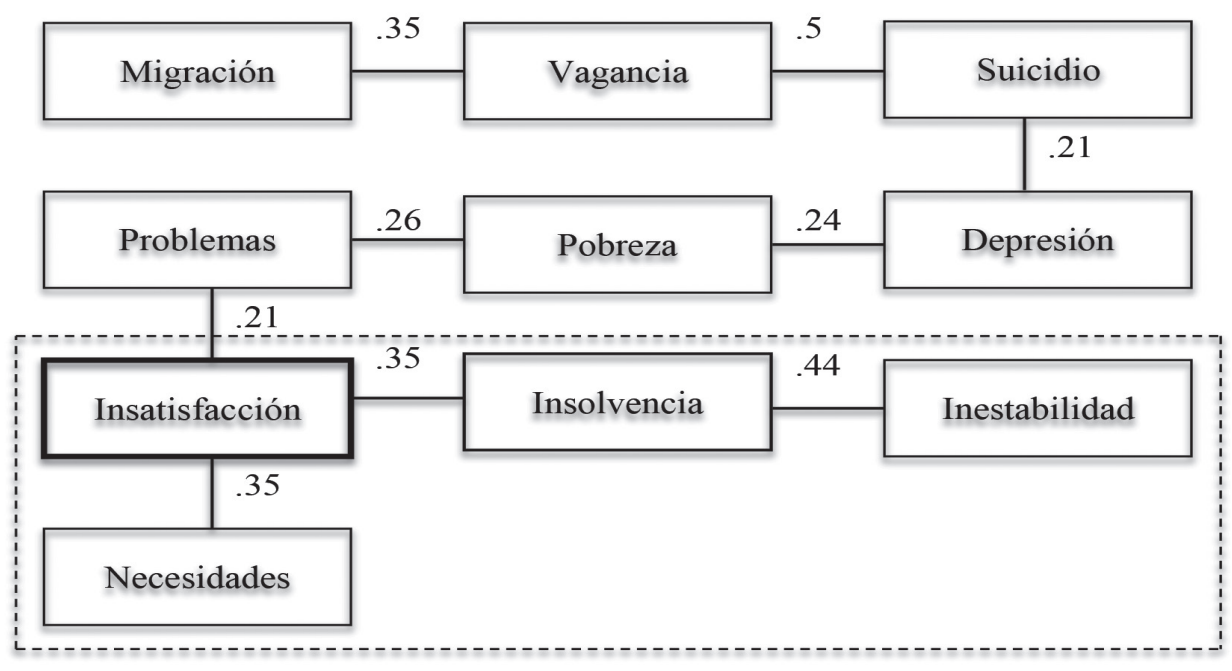




\section{Discusión}

\section{Las RS de empleo: un derecho que requiere esfuerzo y responsabilidad}

El empleo es una ocupación y un derecho que se realiza en organizaciones y empresas a cambio de un salario. Es un trabajo que requiere de experiencia y conocimientos por un lado, así como compromiso, esfuerzo, motivación, responsabilidad, deseos de superación por otro, además de contar con suerte para encontrarlo. Genera estabilidad (emocional y económica), bienestar, autoridad, desarrollo, dignidad e independencia así como la posibilidad de tener futuro, éxitos y logros. Implica la ocupación del tiempo, puede ser agotador y generar insatisfacción cuando no está con acorde a sus necesidades y expectativas. Además, las personas participantes la asocian al desempleo.

Se destaca la centralidad del empleo en las personas participantes, en el sentido de que el empleo no es sólo un factor de producción o una fuerza que impulsa el desarrollo económico, sino que también proporciona identidad, es un medio clave para la integración en la sociedad y es la fuente principal de ingreso y la base de subsistencia (Weller y Roethlisberger, 2012). Se resaltan elementos positivos, tal como lo reporta Pereira (2013), sobre los efectos personales en torno a lograr ser independiente, autónomo, autosuficiente; y por Longo (2003) sobre la autonomía y la independencia generadas.

Para este caso, estas RS de empleo corresponden a las y los jóvenes universitarios, sin embargo, podrían ser diferentes para las y los jóvenes que no tienen acceso al sistema educativo, sobre todo si se encuentran en alguna forma de exclusión económica y social. En un estudio con jóvenes costarricenses en condiciones poco favorables, el empleo era visto como un medio para tener una vida mejor; mientras, para los jóvenes en condiciones de inclusión favorables, el empleo y la profesión son una parte de su identidad en construcción (Beirute, 2015).

Ahora bien, entre los requisitos para desarrollar una actividad laboral se menciona la responsabilidad. Esto fue reportado por Pereira (2013), ya que los jóvenes venezolanos consideran que un buen trabajador debe tener puntualidad, responsabilidad, valores, cumplimiento de normas y horarios, compromiso con la organización, respeto hacia sus superiores y compañeros, y realizar bien su labor. Otro requisito es la importancia de la formación académica, lo que coincide con lo reportado en el estudio ya citado sobre jóvenes costarricenses, pues que para ellos la preparación académica es su principal fortaleza para salir adelante (Beirute, 2015).

Por otro lado, se destaca el elemento de la suerte como un requisito necesario para contar con un empleo. Situación similar al caso de jóvenes mexicanos que consideraban que el empleo es difícil de encontrar (Navarro, 2007). En ambos casos tal parece que en sus RS ubican al empleo como una condición fuera de su control, a esto se le llama: locus de control externo; y consiste en la posibilidad de dominar un acontecimiento según se localice el control dentro o fuera de uno mismo. Se refiere a que no importan los esfuerzos que el individuo haga, el resultado será consecuencia del azar o del poder de los demás (Oros, 2005, p.90).

Llama la atención la inclusión de aspectos negativos en las RS de empleo (es agotador y podría no estar en concordancia con sus expectativas) lo que coincide con lo reportado por estudiantes de bachillerato mexicanos (implica tiempo ocupado, rutina y cansancio) (Torres-López, Acosta y Aguilera, 2017) y en jóvenes universitarios (también mexicanos) con relación a las condiciones de precariedad en que suele desarrollarse (Torres-López y Díaz-Villanueva, 2016), así como por los jóvenes argentinos con relación a los horarios y la duración de la jornada de empleo (Longo, 2003). Además, la mención del término desempleo refuerza la idea de elementos negativos en las RS del empleo. 


\section{Las RS de desempleo: pobreza y precariedad con efectos personales y familiares}

El desempleo es considerado como un tiempo libre, desocupado y desperdiciado que genera pobreza, deudas y precariedad. En lo personal produce: inestabilidad, dependencia, falta de desarrollo, insatisfacción de necesidades; emociones negativas como: ansiedad, depresión, desesperación y angustia. En lo familiar puede provocar problemas como: divorcios y falta de estudio en las hijas y los hijos; en lo social vagancia y suicidio. Además, hay pocas oportunidades de empleo para la población estudiantil. Las formas de afrontamiento al desempleo incluidas por las personas participantes son las de buscar empleo con esfuerzo y humildad, así como migrar a otros países.

Las RS de desempleo de las y los jóvenes participantes coincide con lo reportado por Nascimento y Álvaro (2014) sobre la trayectoria de paso a la edad adulta, la cual se manifiesta como una posibilidad cada vez menos probable para la juventud actual. Debido a que el escenario socio laboral es más complejo, diverso y precario, lo que ocasiona inseguridad e inestabilidad en los ámbitos de su vida social. Por ello las y los jóvenes se encuentran en una situación de inseguridad laboral, entendida como la incapacidad percibida para mantener la continuidad laboral ante una situación de amenaza del trabajo. La situación de desempleo puede implicar, la pérdida o disminución de los recursos económicos y sociales. Además, esta incertidumbre y la ambigüedad con respecto a la continuidad del empleo dificultan, con frecuencia, al trabajador o trabajadora adoptar las estrategias de afrontamiento más efectivas y apropiadas para gestionar esa amenaza (Sora, et al., 2014, p. 16).

En el caso específico de la población de Ecuador, la Encuesta Nacional de empleo, desempleo y subempleo del 2014, reporta que el $43.90 \%$ de las y los desempleados son jóvenes y el $81.37 \%$ se encuentran sin empleo por falta de oportunidades. Lo que lleva a que el $41.19 \%$ de las y los ecuatorianos desempleados tengan falta de superación personal y profesional. Y con relación a la formación académica de éstos, el mayor porcentaje tiene instrucción media (33.97\%) y superior universitaria (21.76\%) (Paredes, Sarmiento, \& Stefos, 2016).

Entre los efectos del desempleo incluidos se encuentran las emociones negativas y como consecuencias sus repercusiones en la salud mental de las y los jóvenes, tal como lo reportaron los estudios con jóvenes mexicanos (Torres-López, et al. 2017; Torres-López, \& Díaz-Villanueva, 2016). En la población de Ecuador, la Encuesta Nacional de empleo, desempleo y subempleo del 2014 ya citada, reporta que el $51.17 \%$ de las y los ecuatorianos desempleados tienen disminución de su autoestima y el 27.92\% de las personas desempleadas ecuatorianos tiene depresión (Paredes, et al., 2016). En un estudio realizado sobre la precariedad laboral y su repercusión en la salud, se encontró que el estrés se relaciona con el desempleo (y en ocasiones con conductas poco saludables como beber alcohol), también se reportan síntomas psicosomáticos, estados de nerviosismo o tensión, pero sobre todo de depresión. Además, una de sus conclusiones es que la precariedad laboral también puede afectar a la salud, porque presenta características similares al desempleo ya que dificulta la posibilidad de construir un proyecto de futuro (Amable, Benach, \& González, 2001).

En cuanto al afrontamiento del desempleo, es una categoría que resulta de vital importancia ya que una de las funciones de las RS es la predisposición a la acción (Jodelet, 2011). Las personas participantes incluyeron una disposición positiva al manifestar la importancia de búsqueda continua de empleo con esfuerzo y humildad. Al respecto Piqueras, Rodríguez, y Rueda (2008) indican que una alta motivación favorece el mantenimiento de conductas de búsqueda de empleo, el interés por la información sobre el mercado de trabajo y la generación de planes o estrategias de actuación para conseguir un puesto de trabajo. La propuesta de afrontamiento menos positiva es la de migrar a otros países en busca de empleo.

\section{Conclusiones}

Las RS sobre los conceptos estudiados muestran una visión compleja y holística, ya que en las RS de empleo y desempleo no sólo se incluyen elementos de tipo económico, sino también los efectos 
personales y sociales que la problemática implica. Se identifica además, una perspectiva complementaria entre los conceptos estudiados, ya que los participantes incluyeron en ambas concepciones elementos positivos y negativos. Con ello, se logra una de las funciones sociales de las RS que es hacer convencionales los objetos, personas y eventos que se encuentran en la vida cotidiana; de tal forma que se establecen maneras de conocimiento distintivas y a la vez compartidas por un grupo cultural determinado (Materán, 2008).

Otra de las funciones de las representaciones sociales es la conformación de identidades personales y sociales, lo que permite la expresión y la configuración de grupos (Materán, 2008). El peligro que se corre es que la crisis del empleo juvenil podría generar en las y los jóvenes, identidades laborales inestables y vulnerables con poco involucramiento y compromiso personal y social.

El conocer las RS de los futuras trabajadoras y futuros trabajadores sobre el empleo y el desempleo, permite acceder a sus conocimientos sobre el tema, así como también a sus experiencias de vida y a la forma en que su contexto ha condicionando sus percepciones y concepciones. Por ello se recomienda considerar las ideas de las y los participantes expresadas en la categoría de afrontamiento positivo (y prevenir las de afrontamiento negativo como es la migración a otros países) para realizar y evaluar programas de promoción para el empleo.

Una de las propuestas que se desprende estos resultados es promover entre las y los estudiantes universitarios opciones como el trabajo colectivo o el auto empleo, aun cuando no son consideradas por ellos. La OIT plantea una forma de superar la crisis del empleo juvenil a partir del desarrollo de iniciativas empresariales propuestas por la misma población juvenil. La promoción de la iniciativa empresarial "abarca toda una serie de actividades realizadas con o sin ánimo de lucro, como el desarrollo de las empresas privadas, el empleo por cuenta propia, las empresas sociales y las cooperativas" (OIT, 2012, p. 13). De acuerdo con Pedraza (2008) en cuanto al apoyo de las iniciativas empresariales juveniles, se debe propender por incentivarlas brindando un respaldo suficiente en dos ejes fundamentales del emprendimiento: la toma de la decisión de ser emprendedor o emprendedora, "teniendo en cuenta la falta de vocación al empleo independiente y la aversión al riesgo y, en segundo lugar, pero no menos importante, el esfuerzo que demanda la constitución de un negocio y su funcionamiento" (Pedraza, 2008, p. 882).

\section{Reflexiones finales}

Convendría ampliar este estudio considerando otros tipos de población, por ejemplo, de jóvenes que no cuentan con formación académica. Máxime cuando se ha observado que el grado de escolaridad está determinando la posición en el empleo y en las ocupaciones de las personas. Se debe priorizar la atención en aquellas personas que deciden no continuar con estudios posteriores a los niveles de primaria, secundaria, media superior y/o carrera técnica, dado que aparecen como los grupos con mayor vulnerabilidad y presentan un mayor crecimiento (Reyes-Terrón \& Elizarrarás-Hernández, 2013). Especialmente, cuando se ha destacado como las condiciones de inclusión y exclusión social podían incidir en la configuración de las visiones sobre el futuro, ya que los resultados del estudio de Beirute (2015) señalan como a mayor inclusión social, mayor desarrollo de capacidades para el ejercicio de libertades.

En cuanto a los aportes del estudio, uno de ellos es presentar la voz de la población juvenil, ya que pocas veces es considerada en el diseño de programas de promoción del empleo, a pesar de ser uno de los actores principales. Otro es la metodología empleada, ya que presenta la ventaja de utilizar técnicas de aplicación y análisis de resultados en forma más rápida que las utilizadas en los estudios sobre representaciones sociales. Por lo cual, la transferibilidad metodológica, citado por Lincoln y Guba (en Flick, 2014), podría resultar de interés en otros campos disciplinares. Finalmente, el enfoque teórico utilizado permite acceder a procesos subjetivos compartidos dentro de un marco cultural, manifestados a través de los discursos y del conocimiento del sentido común. 


\section{Referencias}

Abric, Jean Claude. (2001). Prácticas y representaciones sociales. Ciudad de México, México: Ediciones Coyoacán. Recuperado de: http://www.academia.edu/4035650/ ABRIC_Jean_Claude_org_Practicas_Sociales_y_Representaciones

Aguilera-Guzmán, Rosa María, Mondragón, Liliana, y Medina-Mora, María Elena. (2008). Consideraciones éticas en intervenciones comunitarias: la pertinencia del consentimiento informado. Salud Mental, 31, 129-138. Recuperado de: http://www.scielo.org.mx/scielo.php?script=sci_arttext\&pid $=$ S0185-33252008000200007

Amable, Marcelo, Benach, Joan, y González, Sira. (2001). La precariedad laboral y su repercusión sobre la salud: conceptos y resultados preliminares de un estudio multimétodos. Arch. Prev. Riesgos Labor, 4(4), 169-184.

Banch, María Auxiliadora. (2000). Aproximaciones procesuales y estructurales al estudio de las representaciones sociales. Papers on social representations, 9, 3.1-3.15. Recuperado de: http://www. psych.lse.ac.uk/psr/

Beirute, Tatiana. (2015). Soñando en diferencia: aspiraciones sobre el futuro de juventudes costarricenses. Rev. Reflexiones 94 (1): 23-35.

Carballo-Villagra, Priscilla. (2005). Mercado de trabajo y flexibilidad laboral en las ciencias sociales. Rev. Reflexiones, 84 (1), 33-40.

Doise, Willem, Clemence, Alain, y Lorenzi-Cioldi, Fabio. (1993). The quantitative analysis of social representations. London, Harvester Wheatsheaf.

Flick, Uwe. (2014). La gestión de la calidad en investigación cualitativa. Madrid, Ediciones Morata.

Izquierdo, Tomás, y Alonso, Horacio. (2010). Valores Culturales y Consecuencias Psicosociales del Desempleo en América Latina. Revista de Psicología del Trabajo y de las Organizaciones, 26(2), 123-133. doi: 10.5093/tr2010v26n2a4

Jodelet, Denise. (2011). Aportes del enfoque de las representaciones sociales al campo de la educación. Espacios en Blanco, Serie indagaciones, 21, 133-154. Recuperado de: http://www.scielo.org.ar/ $\mathrm{pdf} / \mathrm{eb} / \mathrm{v} 21 \mathrm{n} 1 / \mathrm{v} 21 \mathrm{n} 1 \mathrm{a} 06 . \mathrm{pdf}$

Longo, María Eugenia. (2003). Representaciones sociales en torno al trabajo e identidad en varones pobres. $6^{\circ}$ Congreso Nacional de Estudios del trabajo. Los trabajadores y el trabajo en la crisis. Asociación Argentina de Especialistas en estudios del trabajo (ASET). Recuperado de: http:// www.csoc.usal.edu.ar/archivos/csoc/docs/idicso-sdti016.pdf

Materán, Angie. (2008). Las representaciones sociales: un referente teórico para la investigación educativa. Geoenseñanza, 13(2), 243-248.

Minayo, Cecilia. (2009). La artesanía de la investigación cualitativa. Buenos Aires, Argentina: Lugar Editorial.

Nascimento, Raquel, y Álvaro, José Luis. (2014). Alargamiento de la juventud e identidad: un estudio de los procesos de transición a la vida adulta de jóvenes en Brasil y España. Athenea Digital, 14(2), 21-37. doi: 10.5565/rev/athenea.1206

Navarro, José. (2007). Experiencias y representaciones sociales del trabajo en jóvenes. En T., Rodríguez, \& M.L., García. (coord.). Representaciones sociales: teoría e investigación (pp. 283-309). Guadalajara, México: Universidad de Guadalajara. Recuperado de: http://www.academia.edu/984196/ Representaciones_sociales_teor\%C3\%ADa_e_investigaci\%C3\%B3n

Oficina Internacional del Trabajo (OIT). (2012). Resolución y conclusiones de la 101. a reunión de la Conferencia Internacional del Trabajo, 2012. La crisis del empleo juvenil: Un llamado a la acción. Ginebra, Suiza: OIT. Recuperado de: http://www.google.com.mx/url?sa $=\mathrm{t} \& \mathrm{rct}=\mathrm{j} \& \mathrm{q}=\&$ esrc $=\mathrm{s} \&$ source $=\mathrm{web} \& \mathrm{~cd}=1 \& \mathrm{ved}=0 \mathrm{CBwQFjAA} \& u r \mathrm{l}=\mathrm{http} \% 3 \mathrm{~A} \% 2 \mathrm{~F} \% 2 \mathrm{Fwww} . \mathrm{ilo}$. org $\% 2 \mathrm{Fwcmsp} 5 \% 2 \mathrm{Fgroups} \% 2 \mathrm{Fpublic} \% 2 \mathrm{~F}---$ ed_norm $\% 2 \mathrm{~F}--$-relconf $\% 2 \mathrm{Fdocuments} \% 2 \mathrm{Fmeeting}$ 
document\%2Fwcms_187080.pdf\&ei=tTFWVZWUF8rHsAWy2oCwBg\&usg=AFQjCNEoiRI3v mMBTGAQcMMIultF1ybVoQ\&bvm=bv.93564037,d.b2w

Oficina Internacional del Trabajo (OIT). (2018). Tesauro de la Oficina Internacional del Trabajo. Biblioteca de la OIT. Recuperado de: http://ilo.multites.net/defaultes.asp

Oros, Laura Beatriz. (2005). Locus de control: Evolución de su concepto y operacionalización, Revista de Psicología de la Universidad de Chile. XIV (1), 89-98. Recuperado de: http://www.redalyc. org/pdf/264/26414107.pdf

Paredes, Ana, Sarmiento, Marisol, y Stefos, Efstathios. (2016). Perfil de los desempleados ecuatorianos. Un análisis multidimensional. Revista PUCE, 102, 325-368.

Pedraza, Aura Cecilia. (2008). El mercado laboral de los jóvenes y las jóvenes de Colombia: realidades y respuestas políticas actuales. Revista Latinoamericana de Ciencias Sociales, Niñez y Juventud, 6(2), 853-884. Recuperado de: http://www.redalyc.org/pdf/773/77360213.pdf

Pereira, Lourdes. (2013). La representación social del trabajo en jóvenes universitarios. Revista Latinoamericana de Derecho Social, 17, 145-177. Recuperado de: http://www.redalyc.org/ pdf/4296/429640272006.pdf

Piqueras, Rafael, Rodríguez, Alberto, y Rueda, Cristina. (2008)._Expectativas y duración del desempleo. Revista de Psicología del Trabajo y de las Organizaciones, 24 (2), 129-151. doi:10.4321/ s1576-59622008000200001.

Pulido-Martínez, Hernán y Carvajal-Marín, Luz Mary. (2013). Riesgos psicolaborales y subjetividad: tensiones contemporáneas entre patologización y despatologización en los discursos psicológicos. Quaderns de Psicologia, 15(1), 69-80. Recuperado de: http://www.quadernsdepsicologia.cat/ article/view/1169

Reyes-Terrón, Ángel Mauricio, y Elizarrarás-Hernández, Moisés. (2013). Los jóvenes y las jóvenes en el Estado de México: sociodemografía y empleo 2010. Revista Latinoamericana de Ciencias Sociales, Niñez y Juventud, 11(1), 287-304. Recuperado de: http://www.scielo.org.co/pdf/rlcs/ v11n1/v11n1a20.pdf

Ruiz, José Ignacio, Ponce De León, Eduardo, Herrera, Ana Nubia, Jiménez, Hugo, y Medellín, Elvers. (2001). Avances en Medición Evaluación en Psicología y Educación. Bogotá, Colombia: Universidad del Bosque.

Sibaja, Irina. (2013). Consideraciones teóricas: la salud sexual y la prevención de infecciones de transmisión sexual en mujeres. Rev. Reflexiones, 92, (2), 79-90.

Smith, Jerome. (1993). Using Anthropac 3.5 and a spreadsheet to compute a free-list salience index. Cultural Anthropology Methods Newsletter, 5(3), 1-3. doi: 10.1177/1525822x9300500301_

Sora, Beatriz, Caballer, Amparo, y Peiró, José María. (2014). La inseguridad laboral y sus consecuencias en un contexto de crisis económica. Papeles del Psicólogo, 35(1), 15-21. Recuperado de: http:// www.redalyc.org/pdf/778/77830184003.pdf

Suri, Harsh. (2011). Purposeful Sampling in Qualitative Research Synthesis. Qualitative Research Journal, 11(2), 63 - 75. DOI 10.3316/QRJ1102063. Recuperado de: http://faculty.yu.edu.jo/Audeh/.../ qualitative\%20paper11.pdf

Torres-López, Teresa M., y Díaz-Villanueva, Ana I. (2016). Representaciones sociales de empleo y desempleo de jóvenes universitarios de una universidad pública de Guadalajara, México. En Norma González \& Laura E. Benhumea. Salud, Trabajo y Género. Jóvenes de cara al trabajo precario. Ciudad de México, México: Editorial Porrúa.

Torres-López, Teresa M., Acosta-Fernández, Martín, y Aguilera-Velasco, María de los Ángeles. (2017). Preparación para el trabajo: Representaciones sociales del empleo y desempleo de jóvenes mexicanos sin trayectoria laboral. Revista Educación y Desarrollo Social, 11(1), 85-101. doi: 10.18359/ reds. 1867 
Vergès, Pierre, Tyszka, Tadeusz, y Vergès, Pierrete. (1994). Noyau central, saillance et propriétés structurales. Papers on Social Representations, 3(1), 3-12. Recuperado de: http://www.psych. lse.ac.uk/psr/

Weller, Jürguen, y Roethlisberger, Claudia. (2012). La calidad del empleo en América Latina: un análisis de conjunto. En S. Farné, [comp.]. La calidad del empleo en el siglo XXI. Bogotá, Colombia: Universidad Externado de Colombia. Recuperado de: http://www.lai.fu-berlin.de/homepages/marull/ Forschung_und.../Farn___2009.pdf 